\title{
Chemical evolution of the Galactic halo and the Origin of Precious Metals
}

\author{
Yuhri Ishimaru ${ }^{1}$, Shinya Wanajo ${ }^{2}$, Wako Aoki ${ }^{3}$, Sean G. Ryan ${ }^{4}$, and \\ Nikos Prantzos ${ }^{5}$ \\ ${ }^{1}$ Academic Support Center, Kogakuin University, 2665-1 Nakano-cho, Hachioji, Tokyo \\ 192-0015, Japan email: kt13121@ns.kogakuin.ac.jp \\ ${ }^{2}$ Research Center for the Early Universe, Graduate School of Science, University of Tokyo, \\ 7-3-1 Hongo, Bunkyo-ku, Tokyo 113-8654, Japan \\ ${ }^{3}$ National Astronomical Observatory of Japan, 2-21-1 Osawa, Mitaka, Tokyo 181-8588, Japan \\ ${ }^{4}$ Department of Physics and Astronomy, The Open University, Milton Keynes, UK \\ ${ }^{5}$ Institut d'Astrophysique de Paris, 98 bis, Boulevard Arago, 75014, Paris, France
}

\begin{abstract}
Observed large scatters in abundances of neutron-capture elements in metal-poor stars may suggest incomplete mixing of the interstellar medium at the beginning of the Galaxy. Comparing predictions by an inhomogeneous chemical evolution model and new observational results with Subaru HDS, we attempt to constrain the origins of $r$-process elements.
\end{abstract}

Keywords. Nuclear reactions, nucleosynthesis, abundances, stars: abundances, Galaxy: evolution, Galaxy: halo

\section{Introduction}

Abundance analysis of metal-poor stars reveals large dispersions in $r$-process elements (e.g., Ryan, Norris, \& Beers 1996, Honda et al. 2004). This may be interpreted as a result of incomplete mixing of the interstellar medium (ISM) at the beginning of the Galaxy. If metal-poor stars contain products of a single or a few supernovae (SNe), huge dispersions in abundances of $r$-process elements possibly imply that their yields are highly dependent on SN progenitor mass. However, no consensus about the origins of $r$-process elements has been achieved, although a few scenarios show some promise (Woosley et al. 1994, Wanajo et al. 2003). In particular, observed enhancement of Sr comparing to $\mathrm{Ba}$ in metal-poor stars suggests the presence of the 'weak' $r$-process which produce mainly lighter $r$-elements. In this study, we discuss the enrichment of $\mathrm{Sr}, \mathrm{Pd}$, $\mathrm{Eu}$, and $\mathrm{Ba}$, using an inhomogeneous chemical evolution model, and attempt to constrain the origin of $r$-process.

\section{Bimodal $r$-process}

In our previous study, the observed wide spread of Eu in metal-poor stars are shown to be well-reproduced by an inhomogeneous enrichment scenario. In particular, sub-solar values of $[\mathrm{Eu} / \mathrm{Fe}]$ in stars of $[\mathrm{Fe} / \mathrm{H}] \sim-3$ can restrict the site of $r$-process as $\mathrm{SNe}$ of low-mass end stars such as $8-10 M_{\odot}$ (cf. Ishimaru et al. 2004). The distribution of the $[\mathrm{Ba} / \mathrm{Fe}]$ abundance ratio also supports this result.

While $\mathrm{Ba}$ and heavier elements seem to fit the solar $r$-process pattern, lighter elements show wide varieties (e.g., Hill et al. 2002, Sneden et al. 2003). In particular, a large dispersion has been found in [Sr/Ba] at low metallicity (e.g., Ryan, Norris, \& Beers 
1996, Honda et al. 2004), suggesting that lighter elements such as Sr does not come from a universal process, which produces $\mathrm{Ba}$ and $\mathrm{Eu}$, but from "weak" $r$-process. As shown in fig. 1, this scenario can well explain the observational distribution of [Sr/Ba], when weak $r$-process produces $\sim 60 \%$ of $\mathrm{Sr}$ but only $\sim 1 \%$ of Ba in metal-poor stars.
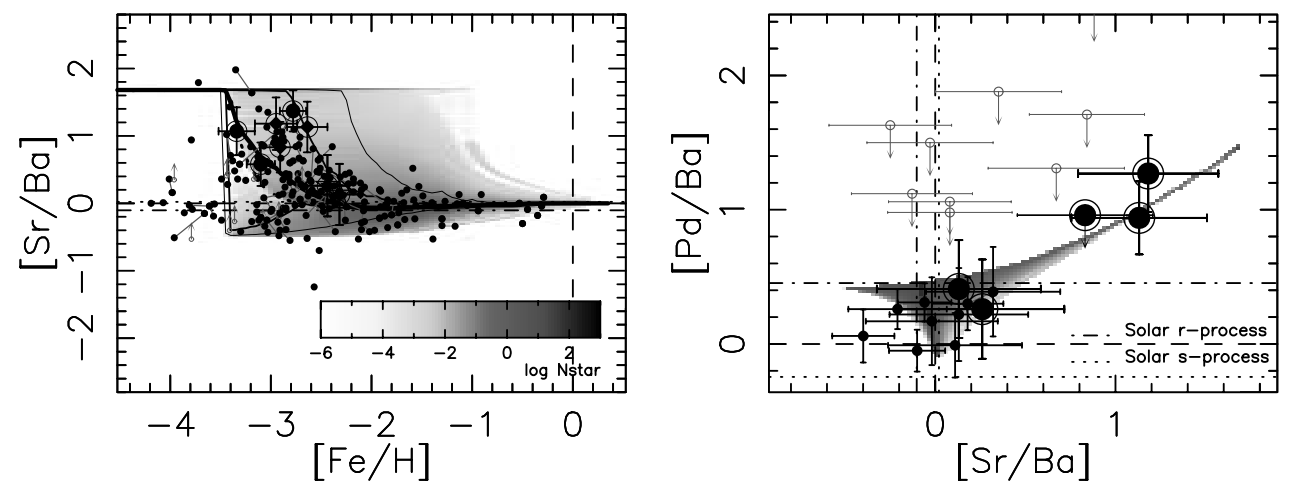

Figure 1. (left panel) $[\mathrm{Sr} / \mathrm{Ba}]$ as a function of $[\mathrm{Fe} / \mathrm{H}]$. Gray-scale indicates predicted distribution of stellar fraction. Weak $r$-process fraction for $\mathrm{Sr}$ and $\mathrm{Ba}$ are assumed as $60 \%$ and $1 \%$, respectively. The average stellar distributions are indicated by thick-solid lines with the $50 \%$ (solid lines) and $90 \%$ confidence intervals (thin-solid lines). The observational data of this study are given by large circles, with other data (small circles).

Figure 2. (right panel) Same with Fig. 1 but for $[\mathrm{Pd} / \mathrm{Ba}]$ vs. [ $\mathrm{Sr} / \mathrm{Ba}]$. Weak $r$-process fraction for $\mathrm{Pd}$ is assumed to be $10 \%$.

\section{Origin of palladium and 'weak' $r$-process}

Intermediate mass elements between $\mathrm{Sr}$ and $\mathrm{Ba}$ must provide clues to understand the nucleosynthesis of weak $r$-process. Therefore, we have estimated $\mathrm{Pd}$ abundance of very metal-poor stars, using Subaru HDS. Fig. 2 shows $[\mathrm{Pd} / \mathrm{Ba}]$ as a function of $[\mathrm{Sr} / \mathrm{Ba}]$. By definition, $[\mathrm{Sr} / \mathrm{Ba}]$ should increase with the fractional contribution of weak $r$-process to the stellar abundances. If $\mathrm{Pd}$ originates from weak $r$ like $\mathrm{Sr},[\mathrm{Pd} / \mathrm{Ba}]$ must show a correlation with a slope of unity to $[\mathrm{Sr} / \mathrm{Ba}]$. If $\mathrm{Pd}$ comes from main $r$ like $\mathrm{Ba},[\mathrm{Pd} / \mathrm{Ba}]$ must be constant. New data show a mild correlation with a slope less than unity, suggesting that the weak $r$-process fraction for $\mathrm{Pd}$ takes intermediate value between those of $\mathrm{Sr}$ and $\mathrm{Ba} ; \sim 10 \%$. Therefore, this result possibly implies that the weak $r$-process fraction decreases with atomic mass from $\mathrm{Sr}$ to Ba. One of the evidence for such nucleosynthesis of weak $r$-process is obtained from our latest result of Subaru observation. The abundance pattern of HD122563 shows over-production in lighter elements around Sr. But the overproduction decreases towards heavier elements (cf. Honda et al., in this proceedings). HD 122563 is possibly enriched by SNe which produce weak $r$-elements.

\section{References}

Hill, V., Plez, B., Cayrel, R., et al. 2002, A\& A, 387, 560

Honda, S., Aoki, W., Kajino, T., et al., 2004, ApJ, 607, 474

Ishimaru, Y., Wanajo, S., Aoki, W., \& Ryan S. G., 2004, ApJ, 600, L47

Ryan, S. G., Norris, J. E., \& Beers, T. C. 1996, ApJ, 471, 254

Sneden, C., Cowan, J. J., Lawler, J. E., et al. 2003, ApJ, 591, 936

Wanajo, S., Tamamura, M., Itoh, N., et al. 2003, ApJ, 593, 968

Woosley, S. E., Wilson, J. R., Mathews, G. J., et al. 1994, ApJ, 433, 229 